Mini Review

\title{
REDOX-MODULATING AND/OR ANTIOXIDANT PROPERTIES OF NIROXIDES - A POTENTIAL REASON FOR DECREASING SIDE-EFFECTS OF CANCER THERAPY
}

\author{
D. Ivanova $^{1^{*}}$, E. Georgieva ${ }^{1}$, D. Lazarova ${ }^{2}$, V. Gadjeva ${ }^{1}$, R. Bakalova ${ }^{2,3}$, Zh. Zhelev $^{1,3,4}$ \\ ${ }^{1}$ Department of Medicinal Chemistry and Biochemistry, Medical Faculty, Trakia University, \\ Stara Zagora, Bulgaria \\ ${ }^{2}$ Medical Faculty, Sofia University, Sofia, Bulgaria \\ ${ }^{3}$ Molecular Imaging Center, National Institute of Radiological Sciences, Chiba, Japan \\ ${ }^{4}$ Institute of Biophysics \& Biomedical Engineering, Bulgarian Academy of Sciences, Sofia, Bulgaria
}

\begin{abstract}
The existing therapeutic approaches - radiation therapy and conventional chemotherapy of cancer, suffer of serious side-effects as a result of increased generation of reactive oxygen and nitrogen species (ROS/RNS) in non-cancer cells and tissues. Currently, the development of oxidative stress in non-cancer tissues of cancer-bearing organism is a serious problem of cancer therapy. It is necessary to ask about a combination of substances expressing a therapeutic synergism as a result of differentiated effects on cancer and normal cells: substances with increased ROS/RNS-scavenging properties targeting normal cells and substances with increased ROS/RNS-generating properties targeting cancer cells. In this context, the redox-modulators are one of the most attractive candidates. The present review outlines the basic principle of this novel therapeutic strategy, focusing on nitroxide derivatives as one of the most promising redox-modulators with antioxidant ability.
\end{abstract}

Key words: Cancer, Oxidative stress, Redox-modulation, Niroxides

\section{Molecular hypothesis of cancer invasion and redox-mediated therapeutic strategy}

One of the widely accepted hypotheses for cancer invasion is based on the assumption that the appearance of malignant cells in the organism can be considered an "inflammatory signal" (Figure 1) (1-3). This leads to a local migration and an activation of a wide variety of immune cells in the microenvironment of the primary tumor locus (termed below as a "target area"). This activation may trigger redox imbalance due to the "oxidative burst" of the immune cells and the production and release of reactive oxygen and nitrogen species (ROS/RNS) in the "target area". In turn, this process will activate the antioxidant defence systems in the "inflamed" region as a compensatory mechanism to prevent oxidative stress in the microenvironment of the primary

\footnotetext{
*Correspondence to: Donika Ivanova, Department of Medicinal Chemistry \& Biochemistry, Medical Faculty, Trakia University, 11 Armejska Str., Stara Zagora 6000, Bulgaria. Tel.0359/883354777; E-mail: donika_georgiewa@abv.bg
}

tumor locus (1-4). The initial redox imbalance and subsequent signal transduction in the "target area" could be a critical regulator of cancer progression. ROS/RNS, produced by the immune cells in the primary tumor locus, could provoke signal transduction in 3 structural (morphological) targets with equal probability: (i) the cancer cells, (ii) the surrounding normal cells, and (iii) the surrounding extracellular matrix.

ROS/RNS have emerged as important mediators of signal transduction that are associated with the activation of the integrin pathway and modulation of integrin function through conformational changes (5). The cross talk between cancer cells and the extracellular matrix also activates integrin-related signal cascades. The oncogenic miRNAs, secreted by cancer cells into the environment, are considered a primary mediator of this process $(6,7)$. The activation of integrin pathway is linked to additional ROS/RNS production by NADPH oxidases, lipoxygenases, mitochondria, etc., leading to a 
vicious cycle $(1,8)$. As a result, the antioxidant defence system crashes with the progression of cancer and tissue redox balance shifts toward
D. IVANOVA, et al.

oxidation, especially in the intermediate/terminal phase (1).

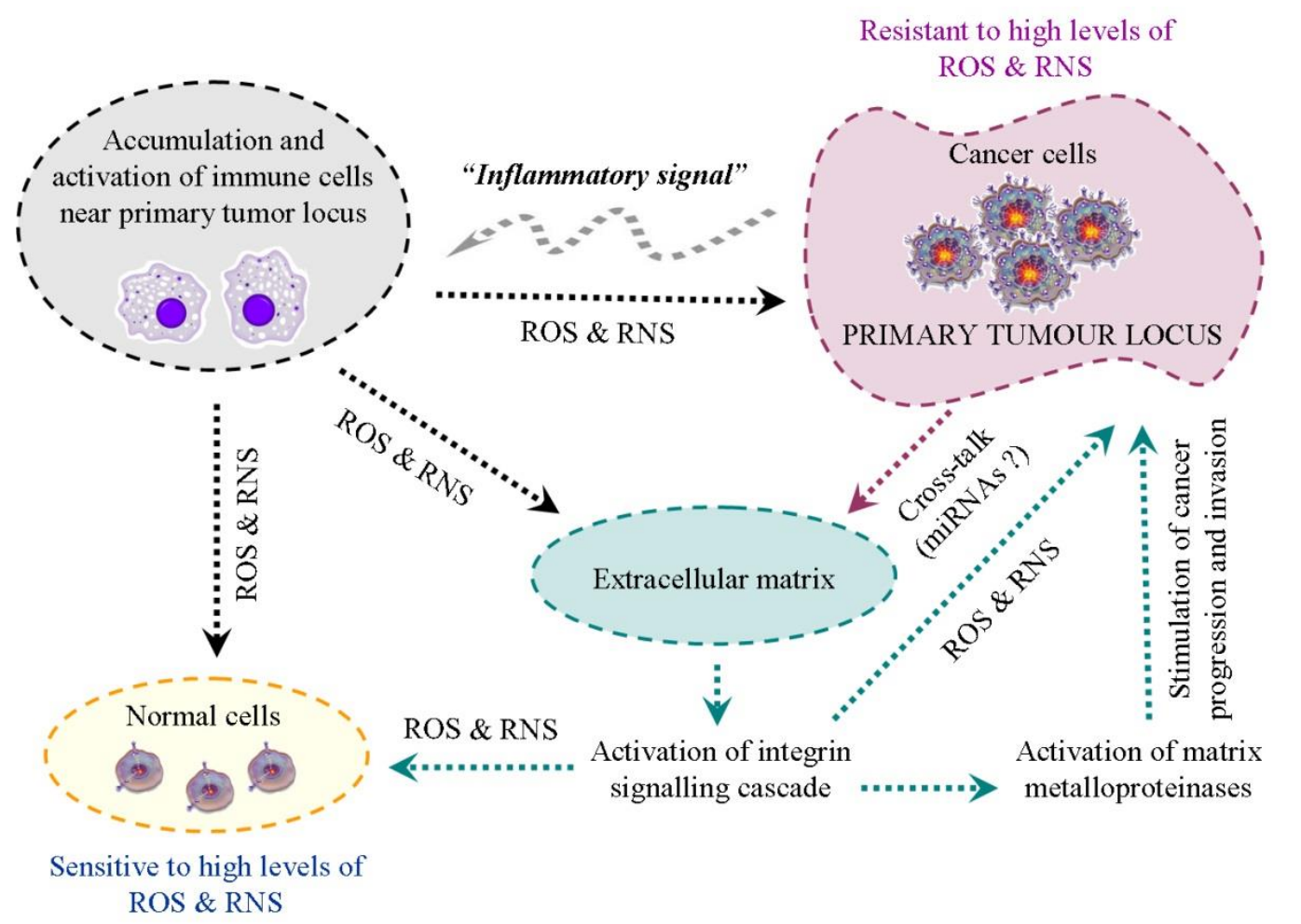

Figure 1. Molecular hypothesis for cancer invasion [according to Bakalova et al. (1)].

Integrin signalling also facilitates cell proliferation and migration, which is intimately linked to the degradation of the extracellular matrix, and activated metalloproteinases (MMPs) are a prerequisite for cancer cell invasion (8). It has been established that the plasma level of MMP9 increases approximately 2 times even in the early stage of cancer development and approximately 3.5 times in the terminal stage (1).

The cancer cells are adapted to the high levels of ROS/RNS and survive. However, the normal surrounding cells and extracellular matrix are not adapted to the abnormal free radical attack and can undergo irreversible changes. Recent studies have shown that antioxidant deficiency develops in tissues distant from the cancer locus of a cancer-bearing organism in the terminal stage (1). These normal tissues become highly sensitive to oxidative damage.

The observations, described above, suggest that the cancer and non-cancer tissues of a cancerbearing organism are equally important therapeutic targets. Obviously, the strong increase of ROS/RNS is the right strategy for killing cancer cells. However, the generation of ROS/RNS has to be localized predominantly in the cancer area to avoid side-effects on noncancer cells and tissues.

The existing therapeutic approaches - radiation therapy and conventional chemotherapy of cancer, suffer of serious side-effects as a result of increased generation of ROS/RNS not only in cancer cells, but also in non-cancer cells and tissues $(4,9,10)$.

Currently, the development of oxidative stress in non-cancer tissues of cancer-bearing organism is a serious problem of cancer therapy. The combination of radiation therapy or standard chemotherapy with antioxidants that increase the protection of non-cancer tissues against oxidative stress should be explored as a therapeutic strategy $(4,10,11)$. However, such strategy would be in conflict with ROS-mediated apoptosis and necrosis in cancer cells. The clinical benefit of using conventional antioxidant 
supplements along with chemotherapy and radiation therapy is highly debatable, and not conclusive. Some of the clinical studies suggest that the antioxidant supplemented patients had a worst survival rate, than the patients who did not use antioxidant supplements $(12,13)$. Although in some cases, use of antioxidant has fewer sideeffects leading to less damage to normal tissues, but with a decrease in the overall survival rate. However, it is also believed that antioxidant supplemented reduction of side-effects depends mainly on using specific anticancer drugs for certain cancers $(9,12-14)$.

It is important to note that the persistent generation of ROS as a result of chemo- and/or radiation therapy can also activate antioxidant adaptive response system and thus to increase the resistance of cancer cells, which will affect negatively the therapeutic outcomes (15). For example, radiation therapy leads directly to ionization of water, then to generation of ROS, which are amplified by mitochondria, generating larger amounts of ROS. Irradiation-induced ROS activate several proliferative and anti-apoptotic pathways (e.g., MAPK pathway, release of VEGF, increase of survivin, etc.), resulting in cytoprotection $(15,16)$.

Therefore, it is necessary to ask about a combination of substances expressing a therapeutic synergism as a result of differentiated effects on cancer and normal cells: substances with increased ROS/RNS-scavenging properties targeting normal cells and substances with increased ROS/RNS-generating properties targeting cancer cells. In this context, the redoxmodulators are one of the most attractive candidates. This new approach is still not clarified and it is a subject to study.

Lipid peroxidation as a major event in radiation therapy and chemotherapy of cancer

The radiation therapy and conventional chemotherapy of cancer are usually accompanied by induction of lipid peroxidation not only in cancer cells and tissues, but also in normal cells and tissues. This process forms the basis of the side-effects.

The lipid peroxidation causes membrane damage as well as oxidative modification of other critical targets (17). The process starts with homolytic fission of the phospholipid hydrocarbon chains. This is a consequence of the attack of ROS, which are able to extract a hydrogen from hydrocarbon chains (LH), leading to formation of an acyl radical ( $\mathrm{L}^{\circ}$ ). All these chemical processes require energy, which can be supplied by radiation, heating, ultrasonication, redox reactions provoked by xenobiotics (e.g., pollutants, toxins and/or drugs), etc. The initially formed acyl radicals react with oxygen and produce acyl peroxyl radicals ( $\left.\mathrm{LOO}^{\circ}\right)$. In turn, acyl peroxyl radicals react with other phospholipid hydrocarbon chains. This mechanism is involved in the propagation phase of lipid peroxidation, leading to formation of hydroperoxides and cyclic peroxides (LOOH) and finally to malonyldialdehyde (MDA), shortchain aldehydes, esters, ketoacids, etc.

The lipid peroxidation could also be started with decomposition of pre-existed lipid hydroperoxides via: (i) homolitic fission (reactions 1 and 2 below); (ii) action of transition metals, even at very low concentration (reaction 3 ); and/or (iii) superoxide anions (reaction 4). The formed alkoxyl radicals ( $\mathrm{LO}^{\circ}$ ) can react with acyl chains producing acyl radicals and alcohol $(\mathrm{LOH})$ (reaction 5), all of them with hydrophobic character (18):

$$
\begin{aligned}
& \mathrm{LOOH} \rightarrow \mathrm{LO}+\mathrm{OH}^{\bullet} \\
& \mathrm{LH}+\mathrm{LOOH} \rightarrow \mathrm{LO}^{\circ}+\mathrm{L}^{-}+\mathrm{OH}^{-}(2) \\
& \mathrm{M}^{\mathrm{n}}+\mathrm{LOOH} \rightarrow \mathrm{LO}^{\circ}+\mathrm{OH}^{-}+\mathrm{M}^{\mathrm{n}+1} \\
& \mathrm{O}_{2}+\mathrm{LOOH} \rightarrow \mathrm{LO}+\mathrm{OH}^{-}+\mathrm{O}_{2} \\
& \mathrm{LO}^{\circ}+\mathrm{LH} \rightarrow \mathrm{L}^{\circ}+\mathrm{LOH}
\end{aligned}
$$

In the cells, lipid peroxidation is controlled by endogenous antioxidant defence system, including antioxidant enzymes [e.g., superoxide dismutase (SOD), catalase, glutathione peroxidase, other peroxidases, etc.] and nonenzymic antioxidants - hydrophilic and hydrophobic (e.g., ascorbate, tocopherols, tocotrienols, reduced glutathione, melatonin, etc.) $(19,20)$. The hydrophilic antioxidants are usually less effective against lipid peroxidation than hydrophobic (lipophilic) antioxidants (19, 20). The regulation of lipid peroxidation in normal range (especially in non-cancer cells and tissues) during cancer therapy is crucial for minimizing side-effects.

\section{Chemical structure of nitroxides and redox- modulating and/or antioxidant capability}

Nitroxide derivatives are one of the most attractive redox-modulators with antioxidant ability due to their free radical scavenging properties and superoxide dismutase-mimetic (SOD-mimetic) effects $(17,21,22)$.

The cyclic nitroxides, also known as aminooxyls or nitroxyls, are stable free radicals 
stabilized by methyl-groups at $\alpha$-position in fivemembered pyrrolidine, pyrroline, or oxazolidine ring structure and six-membered piperidine ring structures (Figure 2). The methyl-groups confer stability to the nitroxide radicals by preventing of radical-radical dismutation and also by limiting the access to reactive substances that can quench the radical species. The substituent groups on the ring (noted by $\mathrm{R}$ ) lead to a diverse range of compounds that can be directed to specific hydrophilic or hydrophobic regions in the cellular microenvironment (17).
Pyrrolidine-type nitroxide

(PROXYL)

Piperidine-type nitroxide

(TEMPO)
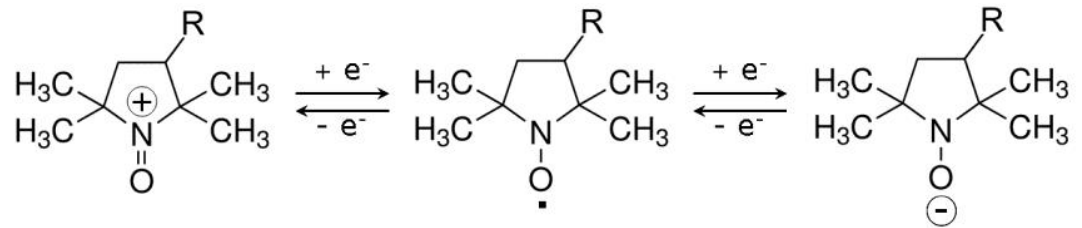<smiles>[R]C1CC(C)(C)[N+](=O)C(C)(C)C1</smiles>

Oxoammonium cation

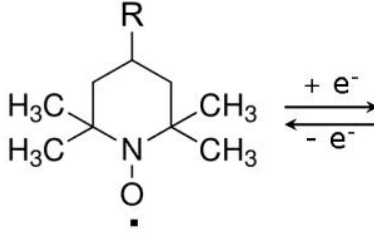

Nitroxide radical

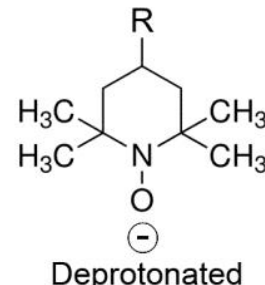

hydroxylamine

Figure 2. Basic chemical structure of nitroxide radicals.

The natural antioxidants and conventional synthetic antioxidants (in their reduced forms) can protect the cells against lipid peroxidation by inhibiting the initiation and retarding the propagation of the free radical chain-reactions. In contrast, the nitroxides (in both radical and hydroxylamine forms) can inhibit lipid peroxidation by participating in chain-reactions at each step $(17,23)$ :

$$
\begin{aligned}
& \mathrm{X}^{\bullet}+\mathrm{RR}^{\prime} \mathrm{NO}^{\circ} \rightarrow \mathrm{RR}^{\prime} \mathrm{NOX} \\
& \mathrm{X}^{*}+\mathrm{RR}^{\prime} \mathrm{NOH} \rightarrow \mathrm{RR}^{\prime} \mathrm{NO}^{\circ}+\mathrm{XH} \\
& \mathrm{L}^{\circ}+\mathrm{RR}^{\prime} \mathrm{NO} \rightarrow \mathrm{RR}^{\circ} \mathrm{NOL}^{\circ} \\
& \mathrm{L}^{\circ}+\mathrm{RR}^{\prime} \mathrm{NOH} \rightarrow \mathrm{RR}^{\prime} \mathrm{NO}^{\circ}+\mathrm{LH} \\
& \mathrm{LO}^{\circ}+\mathrm{RR}^{\prime} \mathrm{NOH} \rightarrow \mathrm{RR}^{\prime} \mathrm{NO}^{\circ}+\mathrm{LOH}
\end{aligned}
$$

It has been reported that nitroxide derivatives, such as TEMPO (2,2,6,6-tetramrthyl-piperidine1-oxyl) and doxylstearic acid, could be used as effective exogenous antioxidants to prevent lipid peroxidation during liposome storage (18). Soule et al. have demonstrated the ability of stable nitroxide radicals to detoxify hypervalent hemecontaining proteins as ferrylmyoglobin $\left(\mathrm{MbFe}^{4+}\right)$, produced in the reaction of metmyoglobin $\left(\mathrm{MbFe}^{3+}\right)$ and $\mathrm{H}_{2} \mathrm{O}_{2}$ (17). The authors evaluate this process by monitoring oxygen evolution, $\mathrm{H}_{2} \mathrm{O}_{2}$ depletion, and redox changes of the heme prosthetic group. It was found that the depletion of $\mathrm{H}_{2} \mathrm{O}_{2}$ can be also enhanced by other nitroxide derivative TEMPOL (4-hydroxy-TEMPO). This study shows that nitroxide (in high concentrations of their radical forms) have a catalase-like activity.

Nitroxides can also prevent generation of $\mathrm{OH}^{*}$ radicals in Fenton reactions by accepting the electron from the reduced metal complexes:

$$
\mathrm{H}^{+}+\mathrm{RR}^{\prime} \mathrm{NO}^{\circ}+\mathrm{Fe}^{2+} \rightarrow \mathrm{RR}^{\prime} \mathrm{NOH}+\mathrm{Fe}^{3+}
$$

It is obvious that nitroxides possess free radical scavenging properties. However, it is necessary to show that these compounds could reduce the levels of oxidative stress and defend the cells against oxidative damage, before to consider them as valuable antioxidants.

Several nitroxides and their corresponding hydroxylamines were tested for protective effects in Chinese hamster V79 lung fibrioblasts, exposed to hypoxanthine/xanthine oxidase under aerobic conditions (17). A large amounts of $\mathrm{O}_{2}{ }^{--}$ and $\mathrm{H}_{2} \mathrm{O}_{2}$ are generated in this reaction and the cells are damaged. TEMPOL and its corresponding hydroxylamine provide a significant protection against cell damage. These results suggest additional modes of protection by nitroxides other than SOD-mimetic activity.

Pyrrolidine- and piperidine-type nitroxides are also very effective redox-modulators due to their reversible conversion between radical form and non-radical forms, participating simultaneously in reactions with oxidizers and reducers (Figure 3) $(17,24)$. In vitro studies indicate that the 
nitroxide radical could be converted rapidly to the hydroxylamine and/or oxoammonium by different cellular compounds (e.g., free ions of transition metals, hydroxyl and hydroperoxyl radicals, ubiquinols, $\mathrm{NAD}(\mathrm{P}) \mathrm{H}$, and ascorbate/dehydroascorbate) $(22,25,26)$. In turn, hydroxylamine and oxoammonium are "superoxide dismutase mimetics" and could restore the nitroxide radical (Figure 3) $(22,25$, 26). The nitroxide and oxoammonium pair acts as an efficient redox couple, which can support
D. IVANOVA, et al.

catalytic processes by reversible one-electron redox reactions. The nitroxide radical/hydroxylamine does not constitute an effective redox couple and it is incapable of supporting catalytic processes. However, the hydroxylamine can function as an efficient hydrogen atom donor and provides antioxidant capabilities as other well-known antioxidants (e.g., ascorbate, tocopherols, tocotrienols, etc.) (17).

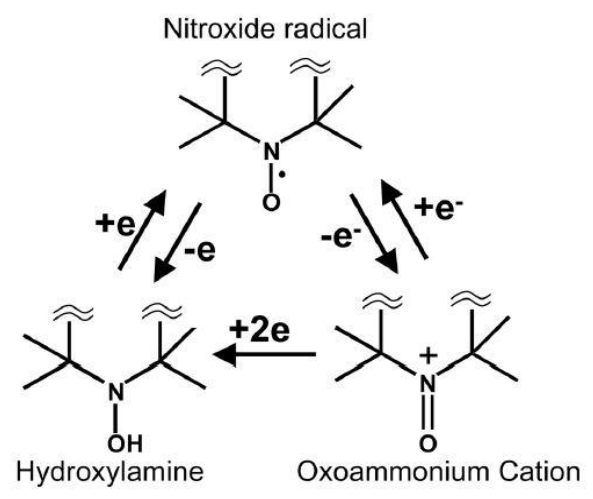

Figure 3. Conversion of nitroxide radical to hydroxylamine or oxoammonium cation in vivo [according to Hyodo et al. (24)].

In vivo studies show that in the living organism the nitroxide derivatives are in equilibrium between the nitroxide radical form and hydroxylamine form, which depends on the oxygen status and redox-status of the tissue milieu (24). It is believed that oxoammonium form is rapidly converted to hydroxylamine form by $\mathrm{NAD}(\mathrm{P}) \mathrm{H}$ in vivo. Thus, the cellular/tissue redox processes convert the compound between the two states - radical and hydroxylamine, and the ratio between these two states is determined by the redox activity within the cell.

Nitroxides as protectors against side-effects, induced by conventional anti-cancer therapy
The protective effect of nitroxides against sideeffects, induced by conventional anti-cancer therapy, has been found about 25 years ago (27). Some nitroxides are considered as selective sensitizers of cancer cells and protectors of normal cells against ionizing radiation and conventional chemotherapeutics, due to the possibility for opposite redox-modulating effects in both types of cells (28). Several theranostic applications of these compounds have been discovered - protection against ionizing radiation, functional imaging of cancer, cancer prevention, anti-obesity effect, etc. (Figure 4) $(1,23,24,29)$.

\section{BIOMEDICAL EFFECTS OF NITROXIDES:}

- Radioprotection

- Chemoprevention and anticancer activity

- Protection against ischemia-reperfusion injury and inflammation

- Protection against neurodegenerative disorders

- Ocular damage protection

- Weight control regulation

Figure 4. Promising biomedical effects of nitroxide derivatives. 
TEMPOL is one of the most widely investigated piperidine nitroxides as redox-modulator, which is currently in clinical trial as radioprotector in cancer radiotherapy (30). TEMPOL is an amphiphilic analog of the highly hydrophobic spin-label TEMPO, which is widely employed in electron-paramagnetic resonance (EPR) spectroscopy. TEMPOL is a stable piperidine nitroxide radical of low molecular weight, which penetrates through biomembranes and scavenges superoxide radicals in vitro and in vivo (31). TEMPO promotes the metabolism of many ROS and improves bioavailability of nitric oxide (21). It is believed that this nitroxide can modulate the level of ROS/RNS and suppress (at least partially) the peroxidation of membrane lipids, denaturation of proteins (including enzymes and ion channels), and to prevent strand-breaks and cross-links in nucleic acids (31).

Various substitutes in the ring structure of the nitroxides allow attachment of target-specific groups. Thus, it is possible to localize the nitroxide molecule in the desired subcellular organelles and to reach a comparatively high concentration in these organelles at very low total doses. Recently, it has been reported a specific localization of nitroxide derivatives (e.g., mito-TEMPO, mito-PROXYL) in mitochondria $(32,33)$. These pyrrolidine and piperidine type nitroxides with alkylphosphonium groups in their rings are localized effectively in mitochondria even when administered at concentration $\sim 3$ orders of magnitude lower than those of non-substituted nitroxides. This suggests that it is possible to use low total doses of these nitroxides and to achieve high local concentrations in mitochondria (or other subcellular compartments), which is very important in anti-cancer therapy. Thus, it is possible to provide more efficient modulation of cellular redox status and redox signaling $(17,32$, 33).

It was shown that the presence of TEMPOradical in antitumor compounds such as 1-(2chlororthyl)-3-cyclohexyl-1-nitrosourea (CCNU) reduces their toxicity and increases radiosensitizing properties, giving a beneficial influence on the antineoplastic properties of this drug $(14,34,35)$. Nitroxide-labeled analogs of CCNU showed lower toxicity and higher anticancer activity in experimental tumor models. Nitroxide-labeled CCNU has a high carbamoylating and low alkylating activity and has a synergistic potential as antitumor agent in comparison with non-labeled drug. Presumably, the low toxicity of the nitroxide-labeled nitrosourea was attributed to the redoxmodulating and/or antioxidant effect of the incorporated nitroxide. The synergistic effect could be due to carbomoylation of thiol groups, which causes changes in the cellular glutathione and endogenous thiols. This strategy has a large potential for sensitizing cancer cells and protecting normal cells and tissues in radiation therapy and chemotherapy.

It was also found that nitroxide-labeled nitrosourea increases the cytotoxic effect of bleomycin and pharmorubicin in human hematological neoplasms, but not in normal lymphocytes (14). Nitroxide labeling could also decrease the toxic side effects of these antibiotics by scavenging superoxide. Thus, the combination of bleomycin (at lower doses) with nitroxide-labeled nitrosourea would decrease bleomycin-induced pulmonary toxicity (14). Several ROS-generating chemical substances are currently undergoing clinical trials as a part of such combined anti-cancer therapy.

Another important modality in the treatment of cancer is the ionizing radiation. In some cases, it is the only option for treatment. In radiation therapy of cancer, it is very important to make a compromise between the highly cytotoxic doses against cancer cells and low cytotoxicity against normal tissues. In this context, it is necessary to apply highly target-specific radiosensitizers for cancer cells - to be able to decrease the radiation dose and to minimize side-effects. This is still very difficult to achieve in clinic. In some cases, it is recommended to use radioprotectors against normal tissues. Any compound which could selectively sensitize hypoxic cells (predominantly within solid tumors) and protect aerated tissues (predominantly normal tissues) may increase the therapeutic gain from radiation and have clinical utility. The nitroxide derivatives seem very promising alternative, because they can serve simultaneously as radiosensitizers for cancer cells and radioprotectors for normal cells and tissues (29). For example, TEMPOL could directly react with and thus detoxify radiolytically produced $\mathrm{OH}^{\circ}$, $\mathrm{H}^{*}$ or aquatic electrons. These products are produced by radiation independently of oxygen and TEMPOL protects the aerobic cells only. TEMPOL may react with $\mathrm{R}^{\circ}$ under hypoxic conditions to result in radiosensitization by producing a non-reversible lesions in cancer (29). It has been established that TEMPOL protects normal cells from lethal doses of 
gamma-radiation with protection factor $\sim 2.5$ in comparison with the non-treated cells (17). These studies show that the nitroxides in both radical and hydroxylamine forms react effectively with free radical species, produced by ionizing radiation. They improve cell survival and protect against DNA double-strand breaks. It is assumed that the higher protective effect of nitroxide radicals is due to their participation in recombination reactions.

The studies on redox-modulating and antioxidant ability of nitroxides suggest that combining radiation therapy and/or standard chemotherapy with low-toxic and cancer-specific redoxmodulators is a promising therapeutic strategy. This approach can increase the protection of non-cancer cells and tissues against oxidative stress, minimize side-effects, and simultaneously to sensitize cancer cells and tissue against ionizing radiation and/or conventional anticancer drugs and to enhance the therapeutic effect.

\section{REFERENCES}

1. Bakalova, R., Zhelev, Z., Aoki, I., Saga, T. Tissue redox activity as a hallmark of carcinogenesis: from early to terminal stages of cancer. Clin Cancer Res, 19:25032517, 2013.

2. Kundu, J.K., Surh, Y-J. Emerging avenues linking inflammation and cancer. Free Radic Biol Med, 52:2013-2037, 2012.

3. Paez, D., Labonte, M.J., Bohanes, P., Zhang, W., Benhamin, L., Ning, Y., Wakatsuki, T., Loupakis, F., Lenz, H-J. Cancer dormancy: a model of early dissemination and late cancer recurrence. Clin Cancer Res, 18:645-653, 2011.

4. Trachootham, D., Alexandre, J., Huang J.P. Targeting cancer cells by ROS-mediated mechanisms: a radical therapeutic approach? Nat Rev Drug Discovery, 9:579$591,2009$.

5. Gregg, D., Carvalho, D.D., Kovacic, H. Integrins and coagulation: A role for ROS/redox signaling? Antioxid Redox Signal, 6:757-764, 2004.

6. Rutnam, Z.J., Wight, T.N., Yang, B.B. miRNAs regulate expression and function of extracellular matrix molecules. Matrix Biol, 32:74-85, 2013.

7. Dalmay, T., Edwards, D.R. miRNAs and the hallmarks of cancer. Oncogene, 25:6170-6175, 2006.
8. Svineng, G., Ravuri, C., Rikardsen, O., Huseby, N.E., Winberg, J.O. The role of reactive oxygen species in integrin and matrix metalloproteinase expression and function. Connect Tissue Res, 49:197-202, 2008 .

9. Ivanova, D., Bakalova, R., Lazarova, D., Gadjeva, V., Zhelev, Z. The impact of reactive oxygen species on anticancer therapeutic strategies. Adv Clin Exp Med, 22:899-908, 2013.

10. Maiti, A.K. Genetic determinants of oxidative stress-mediated sensitization of drug-resistant cancer cells. Int J Cancer, 130:1-9, 2012.

11. Barrera, C. Oxidative stress and lipid peroxidation products in cancer progression and therapy. ISRN Oncology, art. no. 137289, 2012.

12. Lawenda, B.D., Kelly, K.M., Ladas, E.J., Sagar, S.M., Vickers, A., Blumberg, J.B. Should supplemental antioxidant administration be avoided during chemotherapy and radiation therapy? J Natl Cancer Inst, 100:773-783, 2008.

13. Block, K.I., Koch, A.C., Mead, M.N., Tothy, P.K., Newman, R.A., Gyllenhaal, C. Impact of antioxidant supplementation on chemotherapeutic toxicity: A systematic review of the evidence from randomized controlled trials. Int J Cancer, 123:12271239, 2008.

14. Gadjeva, V., Koldamova, R. Spin-labeled 1alkyl-1-nitrosourea synergists of antitumor antibiotics. Anti-Cancer Drug Design, 16:247-253, 2001.

15. Dimova, E.G., Bryant, P.E., Chankova, S.G. "Adaptive response" - some underlying mechanisms and open questions. Genet Mol Biol, 31, 2008.

16. Grdina, D.J., Murley, J.S., Miller, R.C., Mauceri, H.J., Sutton, H.G., Li, J.J., Woloschak, G.E., Weichselbaum, R.R. A surviving-associated adaptive response in radiation therapy. Cancer Res, 73:44184428, 2013.

17. Soule, B.P., Hyodo, F., Matsumoto, K., Simone, N.L., Cook, J.A., Krishna, M.C., Mitchell, J.B. The chemistry and biology of nitroxide compounds. Free Radic Biol Med, 42:1632-1650, 2007.

18. Cimato, A.N., Piehl, L.L., Facorro, G.B., Torti, H.B., Hager, A.A. Antioxidant effects of water- and lipid-soluble nitroxide radicals in liposomes. Free Radic Biol Med, 37:2042-2051, 2004. 
19. Frei, B. (Ed.). Natural Antioxidants in Human Health and Disease, Academic Press, San Diego, 1994.

20. Kagan, V., Packer, L., Serbinova, E., Bakalova, R., Stoyanovsky, D., Zhelev, Z., Harfouf, M., Kitanova, S., Rangelova, D. Mechanisms of vitamin E control of lipid peroxidation: regeneration, synergism, asymmetry, migration and metal chelation.

In: "Biologycal Oxidation Systems" (Reddy, C., Hamilton, G.A., Madyastha, K.M., Eds.), Academic Press, New York, 1990, Vol. 2, pp. 889-908.

21. Wilcox, C.S. Effect of Tempol and redoxcycling nitroxides in models of oxidative stress. Pharmacol Ther, 126:119-145, 2010.

22. Batinić-Haberle, I., Rebouças, J.S., Spasojević, I. Superoxide dismutase mimics: chemistry, pharmacology, and therapeutic potential. Antioxid Redox Signal, 13:877-918, 2010.

23. Likhtenshtein, G.I., Yamauchi, J., Nakatsuji, S., Smirnov, A.I., Tamura, R. Nitroxides: Application in Chemistry, Biomedicine and Material Science, WileyVCH, Weinheim, Germany, 2009.

24. Hyodo, F., Soule, B.P., Matsumoto, K., Matsumoto, S., Cook, J.A., Hyodo, E., Sowers, A.L., Krishna, M.C., Mitchell, J.B. Assessment of tissue redox status using metabolic responsive contrast agents and magnetic resonance imaging. $J$ Pharm Pharmacol, 60:1049-1060, 2008.

25. Matsumoto, K., Hyodo, F., Matsumoto, A., Koretsky, A.P., Sowers, A.L., Mitchell, J.B., Krishna, M.C. High-resolution mapping of tumor redox status by magnetic resonance imaging using nitroxides as redox-sensitive contrast agents. Clin Cancer Res, 12:2355-2362, 2006

26. Matsumoto, A., Matsumoto, K., Matsumoto, S., Hyodo, F., Sowers, A.L., Koscielniak, J.W., Mitchell, J.B., and Krishna M.C. Intracellular hypoxia of tumor tissue estimated by noninvasive EPR oximetry technique using paramagnetic probes. Biol Pharm Bull, 34:142-145, 2011.

27. Mitchell, J.B., DeGraff, W., Kaufman, D., Krishna, M.C., Samuni, A., Finkelshtein, E.,

\section{IVANOVA, et al.}

Ahn, M.S., Hahn, S.M., Gamson, J., Russo, A. Inhibition of oxygen-dependent radiation-induced damage by the nitroxide superoxide dismutase mimetic, Tempol. Arch Biochem Biophys, 289:62-70, 1991.

28. Citrin, D., Cotrim, A.P., Hyodo, F., Baum, B.J., Krishna, M.C., Mitchell, J.B. Radioprotectors and mitigators of radiationinduced normal tissue injury. Oncologist, 15:360-371, 2010.

29. Hahn, S.M., Krishna, M.C., Samuni, A., DeGraff, W., Cuscela, D.O., Johnstone, P., Mitchell, J.B. Potential use of nitroxides in radiation oncology. Cancer Res, 54:2006s2010s, 1994.

30. Metz, J.M., Smith, D., Mick, R., Lustig, R., Mitchell, J., Cherakuri, M., Gratstein, E., Hahn, S.M. A phase I study of topical Tempol for the prevention of alopecia induced by whole brain radiotherapy. Clin Cancer Res, 10:6411-6417, 2004.

31. McDonald, M.C., Zacharowski, K., Bowes, J., Cuzzocrea, S., Thiemermann, C. Tempol reduces infarct size in rodent models of regional myocardial ischemia and reperfusion. Free Radic Biol Med, 27:493 503, 1999.

32. Dikalov, S.I., Kirilyuk, I.A., Voinov, M., Grigor'ev, I.A. EPR detection of cellular and mitochondrial superoxide using cyclic hydrohylamines. Free Radical Res, 45:417430, 2011.

33. Jiang, J., Stoyanovsky, D., Belikova, N.A., Tyurina, Y.Y., Zhao, Q., Tungekar, M.A., Kapralova, V., Huang, Z., Mintz, A.H., Greenberger, J.S., Kagan, V.E. A mitochondria-targeted

triphenylphpsphonium-conjugated nitroxide functions as a radioprotector/mitigator. Radiat Res, 172:706-717, 2009.

34. Sosnovsky, G., Li, S.W. In search for new anticancer drugs. XII. Synthesis and biological evaluation of spin labeled nitrosoureas. Life Sci, 36:1479-1483, 1985.

35. Gadjeva, V., Dimov, A., Georgieva, N. Influence of therapy on the antioxidant status in patients with melanoma. J Clin Pharm Ther, 2008, 33:179-185, 2008. 